polymorphism in the heme oxygenase-1 gene promoter is associated with susceptibility to emphysema. Am J Hum Genet 2000; 66: 187-195.

\section{From the authors:}

We thank D-J. Slebos and colleagues for their comments relating to our paper on the imbalance between haem oxygenase (HO)-1 and inducible nitric oxide synthase (iNOS) in the lungs of severe chronic obstructive pulmonary disease (COPD) patients [1].

Our data showed that expression of HO-1 was decreased in alveolar macrophages in severe COPD, whereas iNOS expression in type- 2 pneumocytes was increased. It is of great interest to see that the results provided by Slebos and colleagues confirm the part of our findings related to HO-1 expression in alveolar macrophages. Since the origin of macrophages retrieved by bronchoalveolar lavage is undetermined, the findings of Slebos and colleagues might indicate that the altered expression of HO-1 in COPD is not limited to the alveolar compartment, but extends to macrophages from the bronchial lumen.

We believe that the presence of current smokers among our patients was a negligible confounding factor for the different expression of HO-1 in severe COPD as compared with control smokers without impairment of lung function [1]. In fact, it was shown in our previous paper [2] that upregulation of HO1 in alveolar macrophages of subjects with smoking history was irrespective of the fact they were current or ex-smokers. The data provided by Slebos and colleagues support the interpretation that HO-1 expression is not influenced directly by cigarette smoke.

We are aware of the potential bias in studies performed on surgically resected specimens, due to the presence of cancer itself or to the selection of the worst part of the lungs in patients who underwent lung volume reduction surgery. However, surgical specimens are precious tissue because they allow direct examination of lung pathology in living patients, which would be otherwise impossible for obvious ethical reasons. This opportunity is particularly relevant in COPD, as peripheral lung is the site where most of the pathological processes take place. This compartment of the lungs cannot be explored using bronchial biopsies. The alternative would be performing post-mortem studies, but in this case clinical and functional characterisation of the subjects is problematic.

One real limitation of immunohistological studies is that they are not suitable for quantitative evaluation of an enzyme such as HO-1. We assumed that the number of positively stained cells is related to enzyme activity, but this may not be true. The attempt to express the results of immunostaining as a score of intensity does not appear to overcome such limitation unless this score is validated by other measurements.

\section{P. Maestrelli}

Dept of Environmental Medicine and Public Health, University of Padova, Padova, Italy

\section{References}

1. Maestrelli P, Paska C, Saetta M, et al. Decreased haem oxygenase-1 and increased inducible nitric oxide synthase in the lung of severe COPD patients. Eur Respir $J$ 2003; 21: 971-976.

2. Maestrelli P, El Messlemani AH, De Fina O, et al. Increased expression of heme oxygenase (HO)-1 in alveolar spaces and HO-2 in alveolar walls of smokers. Am J Respir Crit Care Med 2001; 164: 1508-1513.

\title{
Diagnostic implications of telomerase activity in pleural effusions
}

\section{To the Editor:}

In a recent issue of the European Respiratory Journal, DIKMEN et al. [1] analysed the diagnostic efficacy of telomerase activity in pleural fluid for distinguishing between benign and malignant effusions. They found that the sensitivity and specificity of telomerase activity for diagnosing malignancy was 82 and $80 \%$, respectively. With these data the authors concluded that telomerase activity is a highly sensitive diagnostic biomarker for malignancy and may be used as an adjunct to cytological findings in determining malignant pleural effusions. However, in our opinion, this affirmation can not be applied to clinical practice.

Sensitivity and specificity are important points in the study of the diagnostic value of a test. However, from a clinical point of view, other measures of diagnostic accuracy are of great interest [2], i.e. likelihood ratios and predictive values. Likelihood ratios are the likelihood that a given test result would be expected in a patient with the target disorder compared to the likelihood that the same result would be expected in a patient without the target disorder, and predictive values are the post-test probability $[3,4]$. We calculated these measures using the data reported by DIKMEN et al. [1], and obtained positive and negative likelihood ratios of 4.1 and 0.22 , respectively, which are of modest diagnostic interest [3, 4]. The positive predictive value, in this case, represents the probability that a patient has a malignant pleural effusion if telomerase activity is detected in the pleural fluid. Although it obviously depends on pre-test probability, this predictive value is very low, especially when the prevalence (pre-test probability) is $\sim 20-30 \%$, as is usually observed in general hospitals (table 1) [5]. These findings are similar to those obtained when using classical tumour markers [5].

In our experience [6], the determination of C-reactive protein (CRP) in pleural exudates, which is an inexpensive test and easy to perform, would be of greater interest. A low CRP level $\left(<20 \mathrm{mg} \cdot \mathrm{L}^{-1}\right)$ has a better positive likelihood ratio and positive predictive value for diagnosing malignancy than those of telomerase activity, with the additional advantage that a level $>45 \mathrm{mg} \cdot \mathrm{L}^{-1}$ virtually rules out this possibility.

In conclusion, we consider that the measurement of telomerase activity in pleural fluid adds little to the diagnostic 
Table 1.-Probability (positive predictive value) of malignant pleural effusion when telomerase activity is detected in pleural fluid

Prevalence $\%$ Probability of malignancy $\%$

\section{0}

25

30

40

50

\section{1}

58

64

73

80

work-up of a pleural effusion and should not be used as an adjunct finding in determining malignancy.

\section{E. Garcia-Pachon, I. Padilla-Navas}

Section of Pneumology, Hospital General Universitario, Elche, Alicante, Spain

\section{References}

1. Dikmen G, Dikmen E, Kara M, Sahin E, Dogan P, Ozdemir N. Diagnostic implications of telomerase activity in pleural effusions. Eur Respir $J$ 2003; 22: 422-426.

2. Giard RW, Hermans J. The diagnostic information of tests for the detection of cancer: the usefulness of the likelihood ratio concept. Eur J Cancer 1996; 32A: 20422048.

3. Jaeschke R, Guyatt GH, Sacket DL. Users' guides to the medical literature. III. How to use an article about diagnostic test. B. What are the results and will they help me in caring for my patients? The Evidence-Based Medicine Working Group. JAMA 1994; 271: 703-707.

4. McGee S. Simplifying likelihood ratios. J Gen Intern Med 2002; 17: 646-649.

5. Garcia-Pachon E. Tumor markers for diagnosing malignant pleural effusion? Chest 2002; 121: 302.

6. Garcia-Pachon E, Llorca I. Diagnostic value of C-reactive protein in exudative pleural effusions. Eur J Intern Med 2002; 13: $246-249$. 